Proceedings

\title{
SAMA-VTOL Aerial Image Dataset (SVAID): A New UAV Image Dataset for Advanced Remote Sensing Research ${ }^{+}$
}

\author{
Abbas Ebrahimi 1, *, Mohammad Reza Bayanlou 1, Mehdi Khoshboresh Masouleh ${ }^{2}$ \\ 1 Aerospace Engineering Department, Sharif University of Technology, Tehran, Iran; ebrahimi_a@sharif.ir \\ (A.E.); bayanlom@gmail.com (M.R.B.) \\ 2 School of Surveying and Geospatial Engineering, College of Engineering, University of Tehran, Tehran, \\ Iran; m.khoshboresh@ut.ac.ir (M.K.M.) \\ * Correspondence: ebrahimi_a@sharif.ir; Tel.: +98-021-6616-4632 (A.E.) \\ + Presented at the 2nd International Electronic Conference on Geosciences, 8-15 June 2019;
}

Available online: https://iecg2019.sciforum.net/.

Published: 8 June 2019

\begin{abstract}
SAMA-VTOL aerial image dataset (SVAID) is a new open unmanned aerial vehicles (UAV) image dataset for a wide range of scientific projects in remote sensing (i.e. 3D object modeling, rural/urban mapping, digital elevation/surface model processing, etc.). Open highquality UAV images play an important part in providing and expanding spatial data processing methods. The SVAID includes 120 rural/urban scene images with $80 \%$ overlap between images (forward overlap) and $60 \%$ overlap between flight lines (side overlap) from part of Esfahan province, Iran. The characteristics that make the SVAID an excellent scientific dataset are: (i) very high ground sampling distance (GSD) due to suitable fly height selection; (ii) GNSS-PPK (Post Processing Kinematic) system for improving the spatial accuracy without ground control points (GCPs); (iii) various landscape types (i.e. different types of roofs for commercial/residential buildings, vegetation, etc.), and (iv) uses of the new UAV-photogrammetry platform, named SAMA-VTOL (2019) has been developed by TAREQH Corporation. Additionally, The Agisoft Metashape software was used to analyzing images and produce point clouds, digital surface model (DSM) and orthoimage for evaluating SVAID quality and quantity.
\end{abstract}

Dataset: $\underline{\text { https://doi.org/10.6084/m9.figshare.8216576.v1 }}$

Keywords: SVAID; remote sensing; UAV; SAMA-VTOL; TAREQH

\section{Introduction}

Low-altitude remote sensing or aerial photogrammetry based on unmanned aerial vehicle (UAV) has been widely adopted in many hot fields of science research (i.e. 3D textured modeling of cultural heritage objects and places, affordable and accurate mapping, multi-temporal change detection, agricultural planning, etc.), and it has become a key geospatial data acquisition system [13].

Preparing UAV image datasets and free data sharing, plays an important role in geospatial data analysis and algorithms development [4]. UAV image dataset can be costly due to involvement of the special personnel (i.e. remote sensing specialist), use of expensive equipment (i.e. UAV platform), and providing optimal flight conditions (i.e. weather conditions). Therefore, TAREQH Corporation has produced a dataset with use of a new platform, called SAMA-VTOL aerial image dataset (SVAID). SVAID is a high-quality UAV image dataset for advanced remote sensing research with 
focused on high-precision orthophoto generation and 3D building modeling. Summarizing information about SVAID characteristics is provided in the Table 1.

Table 1. SVAID characteristics.

$\begin{array}{cc}\text { Creating by } & \begin{array}{c}\text { TAREQH Corporation } \\ \text { Thematic categories }\end{array} \\ \begin{array}{c}\text { remote sensing [5], photogrammetry [6], geospatial data } \\ \text { analysis [7], computer vision [8], machine learning [9] } \\ \text { 3D building modeling, point cloud processing, image } \\ \text { mesearch sub-fields }\end{array} & \begin{array}{c}\text { matching, digital elevation/surface model processing } \\ \text { Aircraft }\end{array} \\ \text { SAMA-VTOL (Vertical Takeoff and Landing and fixed-wing) } \\ \text { Fujifilm X-A3 } \\ 6000 \times 4000 \\ \text { Image size (pixel) } \\ \text { Focal length }(\mathbf{c m}) \\ \text { Ground sampling distance } \mathbf{( m m )} \\ \text { Flying altitude }(\mathbf{m}) \\ \text { Date } \\ \text { Location }\end{array}$

\section{Data Description}

\subsection{Original RGB UAV Images}

The original RGB UAV images were captured by SAMA-VTOL are provided for case study. These dataset consist of $120 \mathrm{rural} /$ urban scene images with $80 \%$ forward overlap and $60 \%$ side overlap, where the SVAID uses the WGS 84 (EPSG::4326) coordinate system, as do most GNSS units. A data inventory is provided (Supplementary Material, File 1). Figure 1 shows the study site in the various landscape types with six samples of datasets collected from Esfahan province.

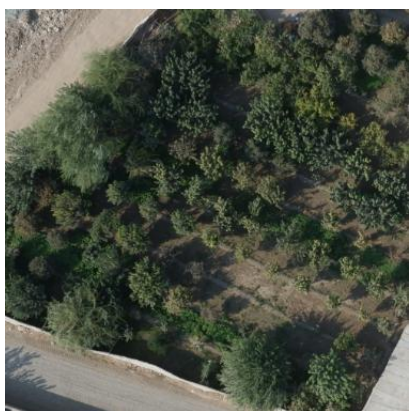

(a)

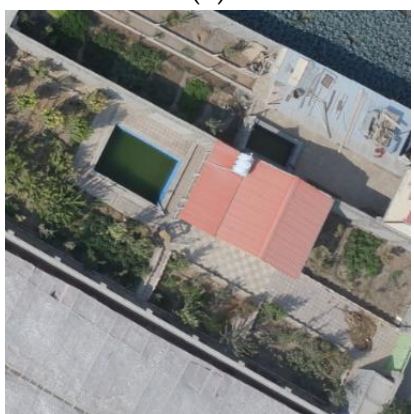

(d)

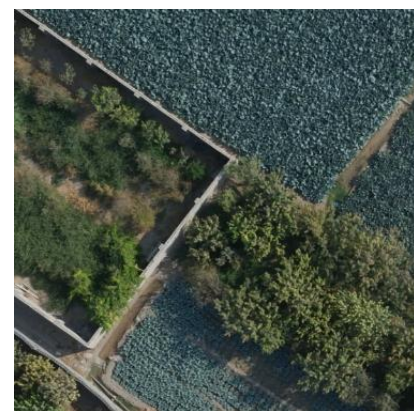

(b)

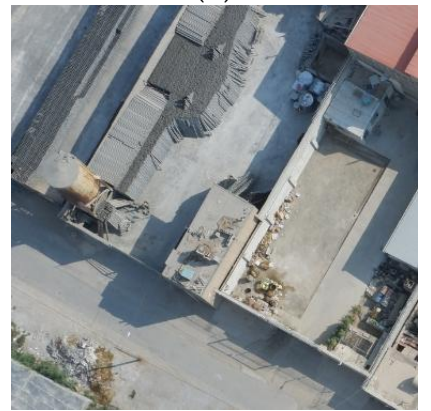

(e)

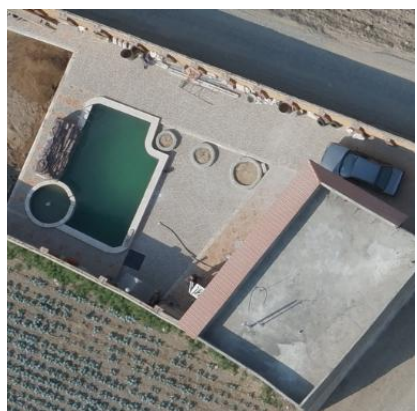

(c)

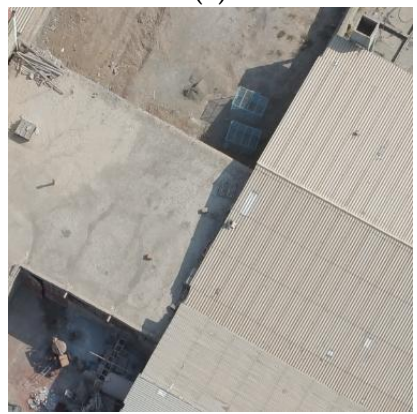

(f)

Figure 1. Various landscape types in SVAID. (a-b) vegetation; (c-d) residential; (e-f) commercial. 


\subsection{Coordinates of Center of Images (CCIs)}

The coordinates of the image center points are provided for each SVAID's images by GNSS-PPK (Post Processing Kinematic) system on the SAMA-VTOL. Figure 2 illustrates the information available in txt file (Supplementary Material, File 2) with each column description given as follows:

First col. Image No: Assigning a unique ID for each image.

Second col. Lat: Latitude.

Third col. Lon: Longitude.

Fourth col. Elevation: Altitude.

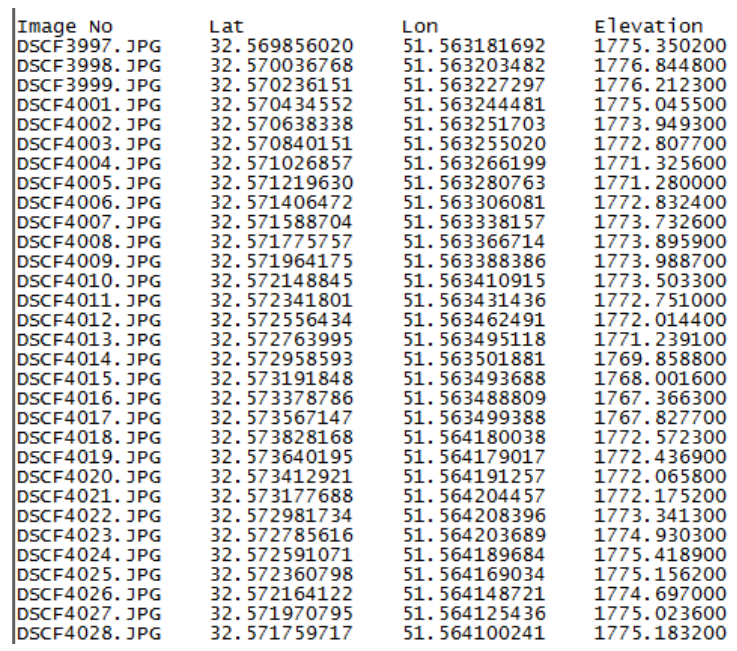

Figure 2. Sample CCIs in txt file.

\section{Methods}

\subsection{Data Collection}

The research site is part of the Esfahan province, Iran (Figure 3). The land cover consists of agricultural land and urban areas.

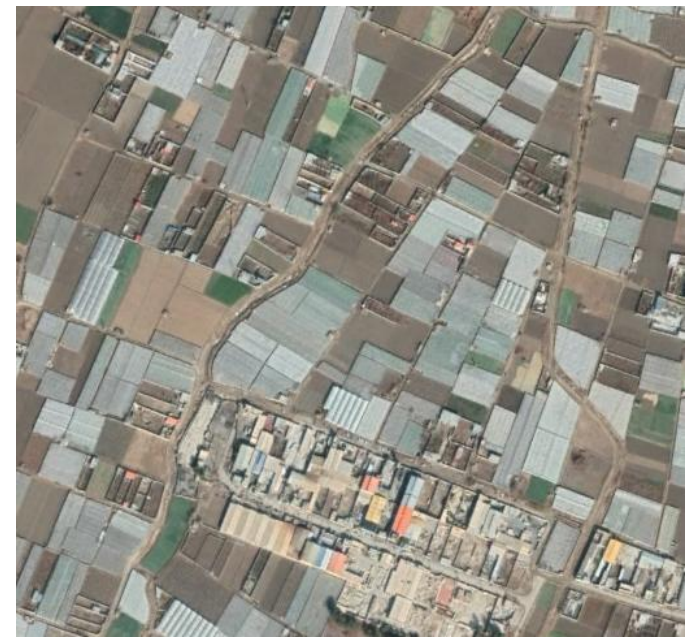

(a)

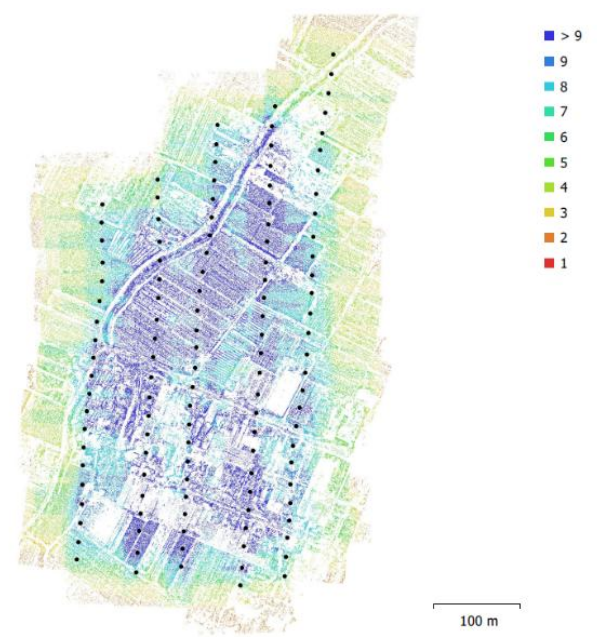

(b)

Figure 3. Google Earth imagery of the study area. (a) Research site; (b) Camera locations and image overlap. 
In this work, SAMA-VTOL was equipped with a Fujifilm X-A3 camera to acquire images (Figure 4). Additionally, the Agisoft Metashape software was used to analyzing images and produce dense point clouds, digital surface model (DSM) and orthoimage for evaluating SVAID quality and quantity and QGroundControl software was used to mission planning and flight control.

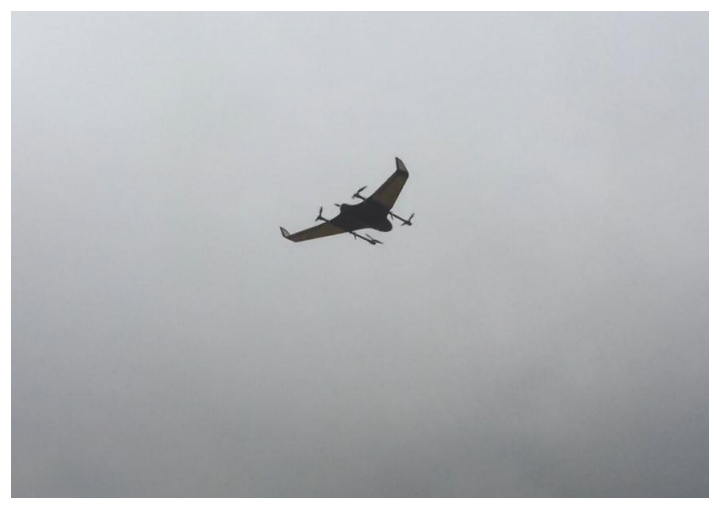

Figure 4. SAMA-VTOL.

\subsection{Data Processing}

The data processing, includes automatic aerial triangulation based bundle block adjustment with camera calibration and model generation by Agisoft Metashape. Figure 5 shows the results of the DSM and orthophoto from the SVAID. Also, Tables 2-3 shows the camera location error and point cloud generated specifications.

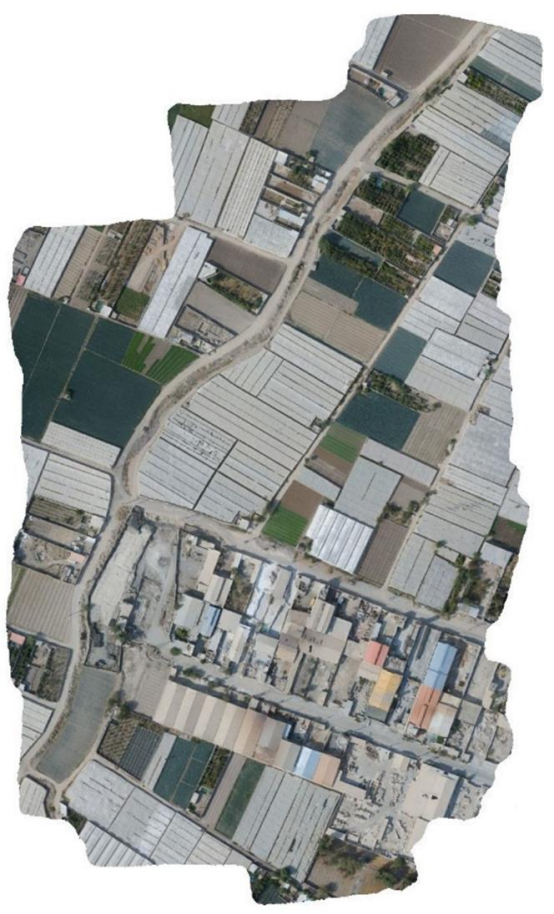

(a)

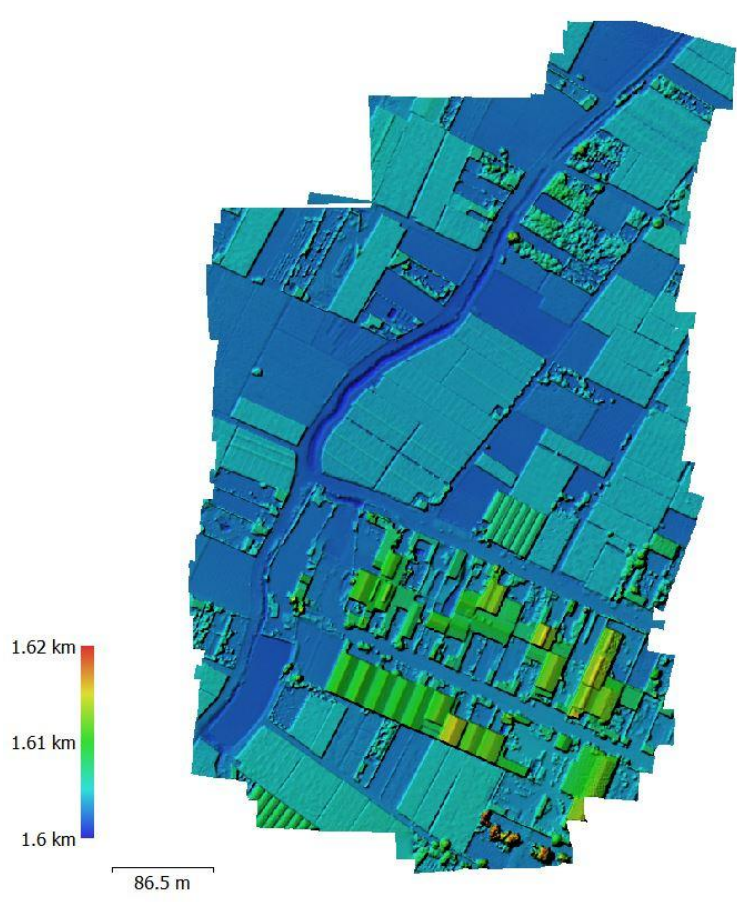

(b)

Figure 5. Products generated by the SVAID. (a) orthomosaic, and (b) DSM.

Table 2. Average camera location error.

\begin{tabular}{ccccc}
\hline $\mathbf{X}(\mathbf{c m})$ & $\mathbf{Y}(\mathbf{c m})$ & $\mathbf{Z}(\mathbf{c m})$ & $\mathbf{X Y}(\mathbf{c m})$ & Total $(\mathbf{c m})$ \\
\hline 1.26172 & 0.33944 & 1.77279 & 1.30658 & 2.20226 \\
\hline
\end{tabular}


Table 3. Point cloud generated specifications.

\begin{tabular}{ccccc}
\hline Points & $\begin{array}{c}\text { RMS reprojection } \\
\text { error (pix) }\end{array}$ & $\begin{array}{c}\text { Max reprojection } \\
\text { error (pix) }\end{array}$ & $\begin{array}{c}\text { Mean key point } \\
\text { size (pix) }\end{array}$ & Point colors \\
\hline 696,962 & 0.501547 & 27.1304 & 4.22775 & 3 bands, uint 8 \\
\hline
\end{tabular}

Author Contributions: Mohammad Reza Bayanlou proposed the conceptual design and building the SAMAVTOL. Mohammad Reza Bayanlou and Mehdi Khoshboresh Masouleh performed the experiments and analyzed the data. Abbas Ebrahimi revised the paper and provided valuable advices for the experiments.

Conflicts of Interest: The authors declare no conflict of interest.

\section{References}

1. Liu, Y.; Zheng, X.; Ai, G.; Zhang, Y.; Zuo, Y. Generating a High-Precision True Digital Orthophoto Map Based on UAV Images. ISPRS Int. J. Geo-Inf. 2018, 7, 333.

2. Akturk, E.; Altunel, A.O. Accuracy assessment of a low-cost UAV derived digital elevation model (DEM) in a highly broken and vegetated terrain. Measurement 2019, 136, 382-386.

3. Kalacska, M.; Lucanus, O.; Sousa, L.; Vieira, T.; Arroyo-Mora, J.P. UAV-Based 3D Point Clouds of Freshwater Fish Habitats, Xingu River Basin, Brazil. Data 2019, 4, 9.

4. Hughes, L.H.; Streicher, S.; Chuprikova, E.; Du Preez, J. A Cluster Graph Approach to Land Cover Classification Boosting. Data 2019, 4, 10.

5. Mathews, A.J. A Practical UAV Remote Sensing Methodology to Generate Multispectral Orthophotos for Vineyards: Estimation of Spectral Reflectance Using Compact Digital Cameras. Int. J. Appl. Geospatial Res. IJAGR 2015, 6, 65-87.

6. Krause, S.; Sanders, T.G.M.; Mund, J.-P.; Greve, K. UAV-Based Photogrammetric Tree Height Measurement for Intensive Forest Monitoring. Remote Sens. 2019, 11, 758.

7. Papakonstantinou, A.; Topouzelis, K.; Pavlogeorgatos, G. Coastline Zones Identification and 3D Coastal Mapping Using UAV Spatial Data. ISPRS Int. J. Geo-Inf. 2016, 5, 75.

8. Carnie, R.; Walker, R.; Corke, P. Image processing algorithms for UAV “sense and avoid." In Proceedings of the Proceedings 2006 IEEE International Conference on Robotics and Automation, 2006. ICRA 2006.; 2006; pp. 2848-2853.

9. Masouleh, M.K.; Shah-Hosseini, R. Fusion of deep learning with adaptive bilateral filter for building outline extraction from remote sensing imagery. J. Appl. Remote Sens. 2018, 12, 1.

(C) 2019 by the authors. Submitted for possible open access publication under the terms and conditions of the Creative Commons Attribution (CC BY) license (http://creativecommons.org/licenses/by/4.0/). 